\title{
Production and Characterization Of Sumac Plantcrystals: Influence of High-Pressure Homogenization on Antioxidant Activity of Sumac (Rhus Coriaria L.)
}

Abraham M. Abraham

Philipps-Universitat Marburg

Camilo Quintero

Universidad de Antioquia

Luis Carrillo-Hormaza

Universidad de Antioquia

Edison Osorio

Universidad de Antioquia

Cornelia M Keck ( $\square$ cornelia.keck@pharmazie.uni-marburg.de)

Philipps-Universitat Marburg https://orcid.org/0000-0001-8888-2340

\section{Research}

Keywords: Plants, Rhus coriaria L., Anacardiaceae, sumac, PlantCrystals, Nano-Nutraceuticals, highpressure homogenization, nanonization, antioxidant capacity, green products, eco-friendly.

Posted Date: February 17th, 2021

DOI: https://doi.org/10.21203/rs.3.rs-233476/v1

License: (c) (i) This work is licensed under a Creative Commons Attribution 4.0 International License.

Read Full License 


\section{Abstract}

- Background: Oxidative stress diseases are usually treated or prevented by using antioxidants from natural or artificial sources. However, as a sustainable source of phytochemicals, plants got a renewed interest in obtaining their active agents using green technologies. Green chemistry is the ability to obtain commercially viable products with desirable properties from widely available renewable sources using eco-friendly methods. The high-pressure homogenization (HPH) technique was introduced into the food industry since it was invented in 1900 to homogenize milk and later to produce fruit juices with a longer shelf-life without preservatives. Recently, HPH was introduced as an eco-friendly method to nano mill plants for improved extraction efficacy without using organic solvents.

-Results: In this study, sumac was used as an antioxidants-rich spice model to investigate the effects of $\mathrm{HPH}$ on its antioxidant capacity (AOC). Sumac was rendered into PlantCrystals by using HPH. Particle size characterization proved the presence of submicron-sized particles (about $750 \mathrm{~nm}$ ). Thus, HPH was able to produce sumac PlantCrystals and increase the AOC of bulk sumac by more than $500 \%$ according to the ORAC (oxygen radical absorbance capacity) assay. The polyphenol and flavonoid contents showed higher values after HPH. Interestingly, the DPPH (1,1-diphenyl-2-picrylhydrazyl) assay also showed a well improved AOC (similar to ascorbic acid) after $\mathrm{HPH}$.

- Conclusion: In fact, in this study, the great potential of the green PlantCrystal-technology could be demonstrated.

\section{Background}

Oxidative stress diseases are considered as one of the serious disorders caused by the imbalance between the free radicals and the antioxidants in the human body [1]. This imbalance is due to the reduced biological system's ability to detoxify the reactive intermediates or repair the oxidative-stress resulted damage. Reactive Oxygen and Nitrogen Species (ROS and RNS, collectively RONS) are responsible for this damage. RONS are reactive derivatives of cellular oxidation processes characterized by the presence of unpaired electrons in their outer shells (i.e. free radicals). Antioxidants are a counterbalance to the reactivity of RONS that work by direct quenching or scavenging of RONS or indirect, by reducing oxidized substrates [2] and stimulating the transcription of other antioxidant systems [3].

Many antioxidants, from natural or artificial sources, are used to counteract RONS. However, an increased, renewed interest in using plants secondary metabolites due to their antioxidative properties is rising again. Hence, new eco-friendly methods need to be developed to introduce plants' phytochemicals in pharmaceuticals, nutraceuticals and cosmetic products.

Green chemistry is the ability to produce viable products with certain properties from widely available sustainable sources (e.g. plants) using eco-friendly methods [4-6]. In particular, there is a great interest in obtaining plant extracts with specific biological properties using green technologies [7-9]. However, there 
is a tension in the use of agriculturally optimum land worldwide to produce chemicals from a biological source and use them in the pharmaceutical industry and personal-care products, versus using these sources in the production of food products for human consumption [10]. Thus, other methods are required to investigate using an eco-friendly method to introduce these sustainable sources (with less amount of starting materials) in the pharmaceutical, nutraceutical, and cosmetic industries.

Plants are a source of bioactive components that are frequently consumed as traditional medicines for generations since they serve as a sustainable source of interesting phytochemicals famous for their antioxidant, anticancer, and antimicrobial activity. These plants are widely consumed in nutrition, but their extracts are prepared using traditional extraction methods, such as infusion, decoction or maceration in aqueous media. To use their non-soluble active compounds in pharmaceutical and personal-care products, their extracts are usually prepared using organic solvents due to their constituents' low solubility in aqueous media [11]. These methods require using solvents and a huge amount of plant starting materials (with the resources for growing, harvesting, transportation requirements, etc.). Therefore, environmentally benign processes that improve sustainability and the eco-friendly production of such products are of high importance and should be developed in an efficient and low-cost manner.

One of these recently used methods to achieve this is high-pressure homogenization (HPH) [12-14]. HPH technique was introduced into the food industry since it was invented by Auguste Gualin in 1900 [15]. It is used for juices to reduce the microbial load, increase juice uniformity and reduce serum separation, while preserving the quality of the fresh juices [15-17]. However, one of the most recent applications of HPH is to nano mill medicinal plants to produce PlantCrystals with improved health beneficial effects $[12,13$, 18]. This allows using the whole plant but destroys all plant cells to ensure a complete release of its phytochemicals (Fig. 1).

PlantCrystals, which are milled plants or parts of plants to sizes below $10 \mu \mathrm{m}$ (Fig. 2), are a novel approach to improve the bio-efficacy of medicinal plants $[12,19]$. PlantCrystals are composed of $100 \%$ plant material in submicron size. The PlantCrystals are obtained by several wet milling procedures, like bead milling, high-speed stirring (HSS), high-pressure homogenization (HPH) or combinations of these methods [12]. Nano milling of plant material allows for complete destruction of all plant cells and thus enables a highly efficient extraction process. In comparison to classical extraction methods, PlantCrystaltechnology does not require the use of organic solvents. In addition, no organic waste is resulted because all plant material remains in the final formulation [12-14].

Hence, the PlantCrystal-technology can be regarded to be a cost-effective, eco-friendly and sustainable production method for herbal medicinal pharmaceuticals and personal-care products. It addresses issues related to improved extraction efficacy, reduction of required plant material, decreased use of organic solvents and the plant material is used completely without producing organic waste $[12,20]$.

Sumac (Rhus coriaria L., Anacardiaceae) is found in Spain, southern Italy, Turkey, and the Middle Eastern countries [10,22-24]. There are more than 250 sumac species in the genus [10]. The fruits are reddish, thin-fleshed drupes covered in some hairs at maturity and form dense clusters at branch tips, sometimes 
called sumac bobs (Fig. 3). All the red fruits of sumac are edible. Acids on hairs on the berries are used to make sumac-ade (a sweetened beverage prepared by soaking and rubbing the sumac drupes in cool water, which is followed by straining the obtained liquid through a tissue). The harvested fruits themselves can be used to make a spice by grinding and sieving. The hard bulb remains after grinding and the fine externally cleaned seeds can be used as an antioxidant-rich spice [23-25]. This has a lemonlike flavor and is often used in Levant cuisine [26, 27]. Sumac fruits are used as dye plants in Morocco in "traditional tanneries of Fez Chouara" that treat and color the leather of animals (Fig. 3). Sumac is also traditionally used in the Middle East, not only as a spice but also as a medicinal plant, e.g., to treat and prevent diabetes, constipated bowel complaints, febrile diseases or dysmenorrhea [24, 28-30].

In the present study, sumac was chosen as a model of an antioxidants-rich spice. Sumac PlantCrystals were produced by using $\mathrm{HPH}$ to improve the release of sumac antioxidants. Hence, the aim of the study was to produce antioxidant-rich and eco-friendly sumac extracts for use in pharmaceutics and cosmetics. The effects of the HPH process on its AOC were investigated and compared with the unprocessed sumac. For this, the dried sumac fruits powder was suspended in a surfactant solution to obtain the bulksuspension. The surfactant solution was used to physically stabilize the produced sumac particles and prevent their agglomeration. Afterward, the coarse, aqueous bulk suspension was first subjected to rotorstator high speed stirring (HSS). This was followed by HPH to obtain the HPH sumac PlantCrystals.

The resulted HPH PlantCrystals were characterized and compared with the bulk and HSS materials regarding size, total polyphenol content (TPC), total flavonoid content (TFC) and antioxidant capacity (AOC). AOC was determined by electron transfer (ET) mechanism using DPPH $\bullet$ (1,1-diphenyl-2picrylhydrazyl), ABTS (2,2'-azinobis (3-ethylbenzothiazoline-6-sulfonic acid)) and FRAP (ferric ion reducing antioxidant power) assays. ORAC (oxygen radical absorbance capacity) assay was also used as a hydrogen atom transfer (HAT) mechanism to determine the AOC.

\section{Results}

The aim of this study was to render sumac bulk material into PlantCrystals by using a combination of HSS and HPH in order to investigate the effect of HPH on sumac AOC using different methods to evaluate the total antioxidant activity of the produced formulations. These different methods can be roughly classified into the following two types: assays based on electron transfer (ET) and assays based on hydrogen atom transfer (HAT) reactions. The total polyphenol and total flavonoid contents were also photometrically determined. Lyophilization-re-dispersion was performed to increase the suspensions' shelf-life. Particle size analysis and DPPH assay were performed on both fresh and lyophilized-redispersed suspensions. All other assays were performed on lyophilized-re-dispersed suspensions.

\subsection{Production and physico-chemical charechterization of HPH sumac PlantCrystals}


Sumac bulk material possessed a mean size of about $200 \mu \mathrm{m}$. HSS reduced the particle size to about 70 $\mu \mathrm{m}$ and HPH could further reduce it to about $10 \mu \mathrm{m}$ (Fig. 4). DLS measurements also proved the presence of submicron sized particles (about $770 \mathrm{~nm}$ ) (Fig. 5).

However, the polydispersity index was very high (about 0.8), indicating a very broad size distribution, which was also confirmed by the LD data (Fig. 4). LD and DLS results also showed that the suspension after $\mathrm{HPH}$ is a mixture of micro- and nanosized particles. Hence, some larger particles remained during the HPH process. However, the number of these particles seems to be very small because light microscopy did not reveal such large particles for the HPH PlantCrystals (Fig. 6). Also, macroscopic observations confirmed a homogeneous distribution of the particles in the final PlantCrystals without any noticed sedimentation (Fig. 7).

Lyophilization (freeze-drying (FD)) converted the produced PlantCrystals into bridle cakes, which were easy to re-disperse in water. Upon re-dispersion of the PlantCrystals, a slight increase in the particle size was detected (Fig. 4). Hence, some of the PlantCrystals formed aggregates that were not fully redispersed. However, DLS data confirmed that the main size population possesses a particle size well below $1 \mu \mathrm{m}$ (Fig. 5). Re-dispersion of the lyophilized bulk and HSS suspensions led to smaller particle sizes. In brief, lyophilization-re-dispersion of PlantCrystals formed some aggregates, but it decreased the particle size of the bulk and micronized HSS suspensions.

The zeta-potential of the PlantCrystals was analyzed in water with a conductivity of $50 \mu \mathrm{S} / \mathrm{cm}$ to detect the charge on the PlantCrystals surface. Based on the zeta-potential measurements before and after lyophilisation-re-dispersion, the obtained values were in the range of $-14 \mathrm{mV}( \pm 1)$ and $-17 \mathrm{mV}( \pm 0.4)$, which classifies the PlantCrystals as mildly stable suspensions.

\subsection{Extraction efficacy and antioxidant capacity}

\subsubsection{Total Polyphenol Content (TPC)}

The TPC value of the suspension obtained after HPH (PlantCrystals) was significantly $(p<0.05)$ increased by $30 \%$ when compared to the bulk and micronized (HSS) materials (Fig. 8). Hence, the available (released) polyphenol content was higher from the PlantCrystals than from the other formulations due to the increased extraction efficacy of the nanosized sumac, caused by the more effective destruction of the plant cells by HPH.

\subsubsection{Total Flavonoid Content (TFC)}


Almost no differences in the TFC values were found between bulk material and the micronized HSSsuspension (Fig. 9). However, the PlantCrystals obtained upon HPH possessed an almost 2-fold higher TFC value (Fig. 9). Hence, the diminution of the plant materials enabled a much better release of the flavonoids than the larger sized material. This difference was not significant.

\subsubsection{Electron Transfer (ET) Assays}

Three different in vitro assays based on electron transfer were used to evaluate the antioxidant activity of sumac formulations produced in this study: scavenging activity on DPPH radicals, reductive power (FRAP) and (2,2'-azinobis(3-ethylbenzothiazoline-6-sulfonic acid)) assay (ABTS). All sumac suspensions showed a propensity to quench the free radicals.

\subsubsection{1. $\mathrm{DPPH}^{\bullet}$ (1,1-diphenyl-2-picrylhydrazyl) assay}

IC50 values represent the amount of active constituents needed to scavenge $50 \%$ of a given amount of free radicals, i.e. low IC 50 values represent a high AOC $[12,13]$. The combination of HSS and HPH techniques could elevate the $A O C$ of the unprocessed bulk-suspension of sumac in a significant way ( $<<$ 0.01 ) (Fig. 10). This indicated an about 3.6-fold increase in the AOC when compared to the bulk suspension (Fig. 10).

The rel. AOC represents the increase or decrease in $A O C$ plant samples when compared to ascorbic acid (standard) or the bulk suspension (Fig. 10). The fresh PlantCrystals from sumac fruits possessed a higher AOC than the ascorbic acid. The fresh bulk suspension possessed an AOC corresponding to about $40 \%$ of the AOC-value of the ascorbic acid. That was followed by an increase of about $90 \%$ and $160 \%$ for the fresh HSS and HPH sumac, respectively (Fig. 10).

Interestingly, also the micro-suspension (HSS-suspension) yielded such high AOC values. So, the reason for the increased $A O C$ values might be due to the destruction of plant cells, which causes an exhausting release of their active constituents. However, lyophilisation-re-dispersion process significantly increased the IC50 values, i.e. reduced the AOC of all the formulations produced in this study (i.e. bulk, HSS and HPH suspensions) (Fig. 10). Nonetheless, lyophilisation-re-dispersion, was an unavoidable step to store the formulations during the study period to perform the other assays, because otherwise the high water content would have caused microbial contamination and consequently destruction of the formulations during storage.

The decrease in the AOC of the lyophilized-re-dispersed HSS-suspension is related to the nature of these active ingredients released, which are more hydrophilic and therefore can be degraded and oxidized rapidly upon re-dispersion in fresh water, which then results in a decrease in $\mathrm{AOC}$ when compared to bulk material or HPH PlantCrystals [12].

Despite the reduction in the AOC upon lyophilization, which was also demonstrated for the PlantCrystals [12], the FD-HPH PlantCrystals showed the best AOC in comparison to the other FD suspensions (Fig. 10). 


\subsubsection{FRAP (Ferric ion Reducing Antioxidant Power) assay}

Bulk-sumac had a similar value compared to the HSS-sumac (Fig. 11). However, HPH could increase the reducing ability of sumac antioxidants and showed an about a $13 \%$ increase in the FRAP value of bulksumac. Thus, the combination of HSS and HPH could significantly $(p<0.05)$ increase the extraction efficiency of sumac antioxidants and led to an elevated AOC (Fig. 11).

\subsubsection{ABTS (2,2'-azinobis(3-ethylbenzothiazoline-6-sulfonic acid)) assay}

The ABTS assay values for sumac formulations ranged from 240 to $260 \mu \mathrm{mol} \mathrm{TE} / \mathrm{g}$ and indicated a good $A O C$ of the produced sumac formulations (Fig. 12). Despite the slight decrease in the ABTS value upon $\mathrm{HPH}$, the final sumac PlantCrystals showed almost similar activity. Thus, almost no or only slight changes were noticed in the sumac evaluated formulations in the ABTS assay. The rel. AOC ${ }_{A B T S}$ was $102 \%$ and $94 \%$ for the HSS suspension and HPH PlantCrystals compared to the bulk sumac suspension. The values of DPPH and FRAP assays showed higher antioxidant activity than ABTS for PlantCrystals (compared to the bulk and HSS suspensions). It is also reported in other studies (for different plant extracts) that ABTS assay showed lower sensitivity than the other ET methods and that the values of the three methods can be varied [31]. Thus, it is important to mention that antioxidant compounds can respond in a different way to different radicals or oxidants, meaning that the use of only one $A O C$ assay can lead to misleading results. Therefore, to obtain a detailed information on the AOC of plant material, different $A O C$ assays must be applied and the results obtained need to be compared to each other (AOC test battery).

Measuring antioxidant capacity by methods such as DPPH, ABTS, and FRAP is characterized by the reduction of free exogenous radicals, such as $\mathrm{DPPH}^{\circ}, \mathrm{ABTS}+^{\circ}$ and the TPTZ-Fe (III) complex, through the transfer of electrons from molecules [32]. Thus, their reaction mechanisms are similar, which partly explains the similarity of the results of these tests. Additionally, in both ABTS and FRAP assay, the redox potential of the antioxidant phytochemicals in the tested formulations is important. The redox potential of $\mathrm{Fe}(\mathrm{II}) /(\mathrm{III})$ and of the redox couple ABTS/ABTS+ are comparable with values of $0.70 \mathrm{~V}$ and $0.68 \mathrm{~V}$, respectively. Thus, these compounds should react in a similar way in both ABTS and FRAP assays, which should lead to a good correlation between their results. This is also clear in our study that almost no or slight changes were noticed between the different formulations (bulk, HSS and HPH) in FRAP and ABTS assays.

\subsubsection{Hydrogen Atom Transfer (HAT) Assays}

\subsubsection{ORAC (oxygen radical absorbance capacity) assay}

ORAC assay was used to gain a better understanding of the total AOC of the produced PlantCrystals from sumac. The ORAC method is characterized by capturing the endogenous radicals that act on oxidized targets and thus is a meaningful tool to express the antioxidant capacity (AOC) of a formulation [12]. In addition, the ORAC assay is considered to be most suitable to assess hydrophilic and lipophilic 
antioxidants at the same time [33]. The ORAC values after micronization (HSS) and after nanosziation $(\mathrm{HPH})$ were 56 and $65 \mu \mathrm{mol} \mathrm{TE} / \mathrm{g}$, respectively.

The AOC-values obtained by the ORAC assay show a similar trend as the values obtained from the DPPH assay and demonstrate a pronounced increase $(p<0.001)$ in the AOC for the PlantCrystals upon HPH, which finally results in a 6.4-fold and 5.6-fold higher AOC when compared to the AOC of the bulk and HSS suspensions, respectively (Fig. 13).

In brief, the AOC of the bulk suspension was found to be $10 \mu \mathrm{mol} T E / \mathrm{g}$, which proved the high antioxidant activity of sumac. However, after micronization (HSS) the ORAC values were $56 \mu \mathrm{mol} \mathrm{TE} / \mathrm{g}$ and after nanonization (HPH) the ORAC values were $64 \mu \mathrm{mol}$ TE/g (Fig. 13).

\section{Discussion}

$\mathrm{HPH}$ was chosen in this study as an eco-friendly technique to investigate its effect on the elevated release of antioxidants from sumac fruits due to the ability of $\mathrm{HPH}$ to disrupt plant cells. Diminution of particles by HPH is based on Bernoulli law [34]. In brief, the plant bulk-suspension is forced to pass through a small orifice that simultaneously leads to a reduction of the static pressure (in the homogenizer gap) below the boiling pressure. This, in turn, leads to the boiling of the fluid and the formation of gas bubbles. Afterwards, the suspension leaves the homogenization gap and normal air pressure conditions are achieved again. This causes the air bubbles to implore immediately. The effect is called cavitation and creates shock waves which then lead to the destruction of particles being suspended in the water phase. Additional forces that occur during HPH and further promote size reduction of the particles include impact and shear forces. As demonstrated, cavitation also causes a reduction in plant particles size and breaks the plant cell walls (Fig. 14). Thus, achieving a pronounced release of plant active ingredients [34]. Adjusting the temperature during the homogenization cycles was an unavoidable step to minimize the possible thermal damage of the materials due to the HPH. This thermal damage can be a result of the friction heat caused due to the high fluid velocity, which elevates the temperature of the suspension about $2-2.5^{\circ} \mathrm{C} / 100$ bar [35].

Sumac PlantCrystals were successfully produced by using the green HPH technique. This led to smaller particle sizes and destroyed the plant cells $[12,13]$. LD data showed that the PlantCrystals had particles in micron size. However, DLS data showed particle sizes well below $10 \mu \mathrm{m}$ (the approximate plant cell size) but had a high Pdl value, indicting the broad size distribution. This board size distribution can be due to the resulted mixture of micronized and nanosized plant particles and the presence of manifold and diverse sumac fruit fragments (for example parts of hairs, pulp, kernels, etc.) that responded differently to the milling forces applied, thus leading to different particle sizes. Freeze-dried and re-suspended PlantCrystals possessed slight difference in sizes, indicating that freeze-drying did not significantly alter the size of the formulations. It led to smaller particle sizes for the unprocessed bulk-suspension and HSS- 
suspension but resulted in bigger particles for the HPH PlantCrystals. This might be a result of slight agglomeration of some individual, nanosized PlantCrystals.

Zeta-potential was analyzed to determine the physical stability of the PlantCrystals. All the produced PlantCrystals (before and after lyophilization-re-dispersion) had values between - $14 \mathrm{mV}$ and $-17 \mathrm{mV}$ in the final formulation indicating decent stability due to steric stabilization of the nonionic surfactant used.

After size characterization and proving the breaking of plant cells of sumac (according to the reduced sizes), the expected release of sumac phytochemicals upon HPH was detected. Therefore, polyphenol and flavonoid contents were determined. The slight increase in the TPC value after applying HSS indicates an increase in the extraction efficacy. However, a more pronounced increase upon HPH was noticed. The increased extraction efficacy of secondary plant compounds from sumac, being mostly antioxidants, resulted in a pronounced increase in the antioxidant capacity. The observed highest TPC value of the PlantCrystals obtained after HPH agreed with the results obtained by Saldo et al. [17] and Schilling et al. [37] (for juices from different materials). This can be related not only to the release of these molecules upon breaking the plant cells but also to the preservation of the phenolic compounds, which can be explained by the HPH impact on the inactivation of polyphenol oxidase enzymes $[35,38]$. However, it has to be stated that the Folin-Ciocalteu method overestimates the TPC due to the lack of selectivity of the Folin-Ciocalteu reagent, which reacts not only with phenols but also with other reducing compounds such as carotenoids, amino acids, sugars and ascorbic acid [39].

In addition to the increased TPC, applying HPH on sumac to obtain its PlantCrystals could improve the total flavonoid content (TFC) due to a pronounced breaking of the plant cells. This is due to the effective forces applied during HPH, such as cavitation, friction, shear and turbulence experienced by the sumac particles of bulk-suspension during the homogenization and thus break the plant cells. Hence, HPH treatment increased the detectable amounts of flavonoid, in more precise words of hesperidin, rutin, apigenin and kaempferol [40].

Furthermore, $\mathrm{HPH}$ increases the flavanone content of the insoluble fractions and this might make them readily available for absorption in the gut. Hence, the insoluble flavanone fractions, which are included in the big particles (inside the intact plant cell), have a reduced absorption rate, i.e., reduced bioavailability. PlantCrystals (after HPH treatment) provide a higher content of flavanones, which increases their bioavailability by increasing their availability for absorption after fermentation by the gut microflora [4144]. In contrast, the unprocessed bulk plant materials are less accessible (in comparison to the nanosized plant materials) to the action of the intestinal microbial enzymes and thus it leads to lower bioavailability [45]. These increased content after HPH of the insoluble flavonoids agreed with our previous findings (for other plants) and with the results published by Velázquez-Estrada et al. (for the orange juice) [16]. Thus, $\mathrm{HPH}$ could nano mill the big particles, improving the extraction efficacy of flavonoids from the bulksuspension, leading to an increase in the flavonoid content and proved that the extraction ability of flavonoids was increased after HPH treatment. 
The application of HSS led to reduced sizes in the upper micrometer level. This leads to a pronounced release of hydrophilic antioxidants. However, HPH technique is even more effective to disrupt the plant cells and cell organelles that host the hydrophobic antioxidants and this results in the release of more hydrophobic antioxidants [12]. Consequently, smaller sizes of the PlantCrystals led to a higher release of these antioxidants.

A significant decrease in the $\mathrm{AOC}$ of the freeze-dried and re-dispersed (in water) formulations was noticed. This might be due to the oxygen contained in the freshwater, which can oxidize the antioxidants - upon the freeze drying and re-dispersion process - thus the more hydrophilic the antioxidants, the more are they prone to the oxidation process. Additionally, the hydrophilic antioxidants in the lyophilized samples get into contact with the re-dispersion medium quicker. Since, the HSS suspension contains more hydrophilic antioxidants than the bulk material [12] - a more pronounced decrease in its AOC upon re-dispersion than the bulk suspension was observed.

In contrast, the PlantCrystals contain both - hydrophilic and lipophilic antioxidants and the hydrophobic antioxidants protect the hydrophilic antioxidants from the oxidation to some extent [12]. Therefore, the all over decrease in AOC upon freezing and re-dispersion is less when compared to the HSS formulation.

Previous studies on sumac extracts' antioxidant potency demonstrated the high antioxidant activity of sumac by using different assays $[24,25,28,40]$. AOC of sumac has been mainly ascribed to gallic acid, ascorbic acid, hesperidin, rutin, apigenin, kaempferol, anthocyanins, hydrolysable tannins, carotenoids, terpenoids and other phenolic compounds $[10,22,28,40]$. In the DPPH assay, the low IC50 value of the bulk sumac indicated its high AOC. The decrease in the IC50 after HSS and HPH to values better than the control (ascorbic acid) can be associated with the destruction of the cellular structure and thus to a higher release of the lipophilic antioxidants from the plant compartments. Hence, the increased availability of insoluble phytochemicals upon HPH, such as carotenoid, hesperidin or kaempferol, increased their detectable AOC values in sumac-PlantCrystals.

For ABTS and FRAP assays, almost no or only slight changes in the AOC were noticed upon the HPH applied on sumac fruits. These two assays are characterized by the reduction of the given free radicals, through the transfer of electrons from molecules $[32,46]$. The redox potential of $\mathrm{Fe}(\mathrm{II}) /(\mathrm{III})$ and of the redox couple $\mathrm{ABTS} / \mathrm{ABTS}+^{\bullet}$ are comparable, which explains their results.

AOC of sumac is ascribed to contain both soluble and insoluble phytochemicals [22]. Therefore, the total $\mathrm{AOC}$ of the formulation is related to the soluble and insoluble active compounds. Despite the previous assays, an additional AOC assay with a different mechanism is necessary to gain a more detailed view of the AOC of sumac PlantCrystals. Therefore, the ORAC assay was also performed. The AOC-values obtained by ORAC assay show a similar trend as the values obtained from the other assays performed in this study and confirm a significant increase in AOC for the PlantCrystals upon nano milling.

The results indicate that the PlantCrystal extraction method (by using HPH) is a two-step process. In the first step, the plant material is milled to sizes in the upper micrometer level. This results in a pronounced 
release of hydrophilic antioxidants, which were more prone to oxidation process after lyophilization-redispersion. In the second step, nano milling of the plant material to sizes $<1 \mu \mathrm{m}$. This leads to the destruction of all plant cells and cell organelles causing an exhausting release of their active constituents. The antioxidative molecules released in the second step are more lipophilic, due to the rupture of the lipophilic cellular compartments that contain these compounds [12].

Nonetheless, the antioxidant activity of phytochemicals is not limited to the free radical scavenging ability and/or oxygen radical absorbance capacity. It also involves the inhibition of the oxidation of lipoproteins in cell membranes, which can be tested using lipid peroxidation inhibition capacity assay. It's recommended that the upcoming studies perform more antioxidant assays to detect the total AOC of sumac PlantCrystals and further identify the specific polyphenolic compounds responsible for the antioxidant activities and study their structure-function interactions in the PlantCrystals formulation.

Nevertheless, the results obtained in this study already support the notion that it is possible to use HPHtechnique as a green technology to crude plant materials and spices to improve their antioxidant activities and enables them to be used in natural pharmaceutical products and cosmetics.

\section{Conclusions}

Sumac fruit PlantCrystals were successfully produced by the combination of HSS and HPH. The bulksuspension of sumac possesses high antioxidant activity, which was further improved by the nanonization process, i.e., the obtained sumac PlantCrystals demonstrated the highest antioxidant activity. Lyophilization, when compared to freshly produced formulations, reduced the AOC of sumac bulk suspensions and PlantCrystals, but was necessary to avoid microbial instability of the formulations during storage. However, also in the lyophilized samples the AOC of the sumac PlantCrystals was improved when compared to the bulk material. In fact, the PlantCrystal-technology was successfully exploited to produces highly effective and eco-friendly plant extracts from sumac fruits that can be used in natural personal-care products and cosmetics.

The results also demonstrated that the improved $\mathrm{AOC}$ upon $\mathrm{HPH}$ is due to the effective release of plant phytochemicals caused by the efficient disruption of the plant cells. The principle is simple, and it can be assumed that it can also be exploited for other plants and parts of plants. PlantCrystals increase bioefficacy of the PlantCrystal extracts. Hence, less plant material is required for its production. This reduces costs for planting, growing, harvesting and transportation of plants. In addition, PlantCrystals produce no organic waste, because all plant material remains in the final formulation. Finally, they are produced without organic solvent. This means the PlantCrystal-technology produces highly effective and ecofriendly plant extracts at the same time. With this the PlantCrystal-technology is a powerful and superior formulation principle for the formulation of plant derived medicinal, nutraceutical or cosmeceutical products when compared to classical plant extracts (e.g., infuses, macerates, etc.).

\section{Materials And Methods}




\subsection{Production of sumac PlantCrystals}

Dried and ground sumac fruits were bought from a local market in Nablus, Palestine. The bulk material was further grinded using mortar and pestle, a hand blender (Elta Lizenz $\mathrm{GmbH}$, Oststeinbeck, Germany) and an AR1105 electrical grinder (Moulinex, Grenoble, France). In the next step, the bulk powder was suspended in a surfactant solution of polysorbate 80 (Tween $80^{\circledR}$, Sigma-Aldrich, Darmstadt, Germany) $1 \%(\mathrm{w} / \mathrm{w})$ to obtain the bulk suspension of a final concentration of $1 \%(\mathrm{w} / \mathrm{w})$ sumac fruit. Subsequently, the bulk-suspension was pre-milled using high speed stirring (HSS) by a rotor-stator mixer (Ultra Turrax T25, IKA, Königswinter, Germany) employing different rotation speeds to obtain what was called a HSS or micro-suspension. The micro-suspension obtained was subjected to the nano milling process using highpressure homogenization $(\mathrm{HPH})$ with a LAB 40 piston gap homogenizer in discontinuous mode with a batch size of $40 \mathrm{~mL}$ (GEA Soavi, Lübeck, Germany) at 1500 bar for up to 30 cycles to yield the PlantCrystals [12]. A water bath was used to avoid the possible thermal damage of the sumac phytochemicals during $\mathrm{HPH}$ with a temperature set to $5^{\circ} \mathrm{C}$ [47].

PlantCrystals are prone to microbiological contamination. To improve the suspensions' shelf life and use them in the following experiments, all the formulations were lyophilized directly after production.

Lyophilisation was performed using an Alpha 1-4 LSC lyophilizer (Martin Christ Gefriertrocknungsanlagen $\mathrm{GmbH}$, Osterode am Harz, Germany). The samples were frozen at $\left(-80^{\circ} \mathrm{C}\right)$ overnight, then main dried $(-50$ $\left.{ }^{\circ} \mathrm{C}, 0.120 \mathrm{mbar}\right)$ for 48 hours and the final drying $\left(25^{\circ} \mathrm{C}, 1 \mathrm{mbar}\right)$ was performed for 24 hours. Mannitol $20 \% \mathrm{w} / \mathrm{v}$ was used as a cryoprotectant [12]. Lyophilized samples were homogenized using mortar and pestle and then re-dispersed in purified water directly before analysis to obtain $1 \%(\mathrm{w} / \mathrm{w})$ plant material.

\subsection{Physico-chemical characterization of sumac PlantCrystals}

In-process and at the end of the production particle sizes and degree of agglomeration of bulk material, HSS and HPH suspensions were characterized using a combination of three methods of particle size characterization on the same day of the production and/or re-dispersion. This includes light microscopy equipped with SC50 CMOS color camera (Olympus soft imaging solutions GmbH, Münster, Germany), dynamic and static laser light scattering (Zetasizer Nano ZS and Mastersizer 3000, Malvern-Panalytical, Kassel, Germany). Dynamic light scattering (DLS) results show the hydrodynamic diameter (z-average) and the polydispersity index (Pdl); as an indication of the width of the size distribution. Static laser light scattering (SLS), or what is also called laser diffraction (LD), was performed to detect the possible large particles that can remain after HPH. Mie-theory with optical parameters set to 1.45 (real refractive index) and 0.01 (imaginary refractive index) was used for the LD analysis. Sonication was avoided during the measurements to evade any resulted breaking of the possible agglomerates [48].

Zeta-potential (ZP) was measured at $20^{\circ} \mathrm{C}$ by using Zetasizer Nano ZS (Malvern-Panalytical, Kassel, Germany). To measure the ZP, the electrophoretic mobility was determined with Laser-Doppler- 
anemometry (LDA). Then Malvern Zetasizer software converted the electrophoretic mobility into the ZP by using Helmholtz-Smoluchowski equation. The measurements were performed in conductivity adjusted purified water $(50 \mu \mathrm{S} / \mathrm{cm})$. The analysis was performed in triplicates and shown as an average \pm the mean standard deviation (SD).

\subsection{Extraction efficacy and antioxidant capacity}

\subsubsection{Total Polyphenol Content (TPC)}

TPC was measured by the Folin-Ciocalteu method $[46,49,50]$. The reaction mixture contained purified water, sample or standard, $20 \%$ sodium carbonate (Merck Chemicals, Darmstadt, Germany), and 2N Folin-Ciocalteu reagent (Merck Chemicals, Darmstadt, Germany) at a ratio of 7.5:1:1:0.5. After one hour at room temperature and with protection from light, the absorbance was measured at $760 \mathrm{~nm}$ using a UV/Vis spectrophotometer (BioTek Instruments, Winooski, USA). Then results were expressed in mg gallic acid (Sigma-Aldrich Chemical Co., Louis, MO, USA) equivalents per gram of sample (mg GAE/g) based on a calibration curve with gallic acid $(10-100 \mu \mathrm{g} / \mathrm{mL})$. In addition, results were expressed as relative extraction efficacy of these phenolic compounds in comparison to the non-processed bulk material.

\subsubsection{Total Flavonoid Content (TFC)}

TFC was determined using a method being based on the interaction of flavonoids with $\mathrm{AlCl}_{3}$ (Merck Chemicals, Darmstadt, Germany) that leads to the formation of a complex that can be determined via a UV/Vis spectrophotometer (BioTek Instruments, Winooski, USA) at $420 \mathrm{~nm}$ [49]. The TFC values are expressed as mg rutin (Cayman Chemical Co., Michigan, USA) equivalents (RE) per gram of suspension based on a calibration curve with rutin $(10-100 \mu \mathrm{g} / \mathrm{mL})$. In addition, results were expressed as relative extraction efficacy and compared to the non-processed bulk material.

\subsubsection{Electron Transfer (ET) Assays}

\subsubsection{1. $\mathrm{DPPH} \bullet$ (1,1-diphenyl-2-picrylhydrazyl) assay}

The antioxidant capacity of the fresh and lyophilized sumac PlantCrystals was investigated using the $\mathrm{DPPH}^{\bullet}$ (1,1-diphenyl-2-picrylhydrazyl, Sigma-Aldrich Chemie GmbH, Steinheim am Albuch, Germany) assay according to the method proposed by Sharma and Bhat $[12,14,50,51]$ and compared to AOC of the HSS-suspension and bulk materials, where ascorbic acid was used as standard. The test was performed in a 96-well plate. Initially, a $0.2 \mathrm{mM}$ DPPH solution was prepared in methanol. Afterward, dilution of each sample was done using distilled water and finally $100 \mu \mathrm{L}$ of DPPH solution was added. Methanol in addition to distilled water were used as blanks. That was followed by incubating the plates in the dark for 30 minutes to allow the reaction to occur. In the next step, the absorbance was measured by a Multi-plate UV/Vis spectrophotometer at a wavelength of $517 \mathrm{~nm}$ by using Multiskan GO (Thermo 
Scientific, Dreieich, Germany). The assay was performed in triplicate and then the percentage of radical scavenging activity (RSA) values were calculated from the following equation:

$$
R S A(\%)=\left(\frac{\text { absorbance }_{D P P H}-\text { absorbance }_{\text {sample }}}{\text { absorbance }_{D P P H}}\right) \times 100
$$

DPPH results were determined by the IC $50 \%$ and as a relative antioxidant capacity compared to the nonprocessed bulk sumac.

\subsubsection{FRAP (Ferric ion Reducing Antioxidant Power) assay}

This method evaluates the capacity of the antioxidants in the samples to reduce the ferric ion $\left(\mathrm{Fe}^{3+}\right)$ in an acidic medium in the presence of TPTZ (2,4,6-Tris(2-pyridyl)-s-triazine) to form the ferrous form $\left(\mathrm{Fe}^{2+}\right)$. This reaction leads to a reduced TPTZ-Fe (III) complex with blue color, measured at $593 \mathrm{~nm}$. The working solution contained $300 \mathrm{mM}$ acetate buffer (pH 3.6), a 40 mM TPTZ solution (Sigma-Aldrich Chemical Co., Louis, MO, USA), and a $20 \mathrm{mM} \mathrm{FeCl}_{3} \cdot 6 \mathrm{H}_{2} \mathrm{O}$ solution (Merck Chemicals, Darmstadt, Germany) in water at a 10:1:1 ratio. Suspensions and the working FRAP solution were mixed at a 1:25 ratio for $10 \mathrm{~min}$ at $37^{\circ} \mathrm{C}$ in a dark place. The absorbance was taken at $593 \mathrm{~nm}$ using a UV/Vis spectrophotometer (BioTek Instruments, Winooski, USA) [46]. A calibration curve with Trolox (Sigma-Aldrich Chemical Co., Louis, MO, USA) was used. The results are expressed in $\mu$ mol Trolox equivalents per gram of suspension and relative antioxidant capacity compared to the non-processed bulk material.

\subsubsection{ABTS (2,2'-azinobis(3-ethylbenzothiazoline-6-sulfonic acid)) assay}

In the ABTS assay, the greenish-blue stable radical cation $\mathrm{ABTS}^{\circ+}$ (2,2'-azinobis(3-ethylbenzothiazoline-6sulfonate) is produced by oxidation and has an absorbance maximum at $734 \mathrm{~nm}$. The absorbance was measured using a UV/Vis spectrophotometer (BioTek Instruments, Winooski, USA). ABTS ${ }^{\circ+}$ was generated by the reaction of $7 \mathrm{mM}$ ABTS (Sigma-Aldrich Chemical Co., Louis, MO, USA) and $2.45 \mathrm{mM}$ potassium persulfate (Merck Chemicals, Darmstadt, Germany) in PBS ( $\mathrm{pH}: 7.4$ ) in a dark place at room temperature for $16 \mathrm{~h}$. ABTS values are expressed in $\mu$ mol Trolox (Sigma-Aldrich Chemical Co., Louis, MO, USA) equivalents per gram of sample $(\mu \mathrm{mol} \mathrm{TE} / \mathrm{g})$. The calculations were based on a Trolox calibration curve vs. the inhibition percent of the radical ABTS ${ }^{*+}$. The re-dispersed samples were added after ABTS ${ }^{\circ+}$ has been generated [46]. The data were also expressed as relative antioxidant capacity in comparison to the non-processed bulk material.

\subsubsection{Hydrogen Atom Transfer (HAT) Assays}

5.3.4.1. ORAC (oxygen radical absorbance capacity) assay 
AAPH (2,2'-azobis(2-methylpropionamidine) dihydrochloride, Merck Chemicals, Darmstadt, Germany) was used as a peroxyl radical generator, fluorescein (Merck Chemicals, Darmstadt, Germany) was used as fluorescent and Trolox (6-hydroxy-2,5,7,8tetramethylchromane-2-carboxylic acid, Merck Chemicals, Darmstadt, Germany) was used as a standard. Fluorescein intensity was measured every minute for 2 hours at excitation and emission wavelengths of 485 and $520 \mathrm{~nm}$, respectively. ORAC values were expressed as $\mu \mathrm{mol}$ Trolox equivalents per gram of suspension $[46,52,53]$.

\subsubsection{Statistical analysis}

All results were expressed as mean \pm SD. All statistical analyses were performed using GraphPad Prism 5 (GraphPad Software Inc., San Diego, CA, USA). Analysis of variance and Tukey's multiple comparison tests were performed to evaluate significant $(p<0.05)$ differences between bulk-materials, HSS and HPH PlantCrystals.

\section{Declarations}

- Ethics approval and consent to participate: "Not applicable"

- Consent for publication: "Not applicable"

- Availability of data and materials: "All data generated or analysed during this study are included in this published article"

- Competing interests: "The authors declare that they have no competing interests"

- Funding: "This research received no external funding."

- Author Contributions: "Conceptualization, A.M.A. and C.M.K.; methodology, A.M.A. and C.Q.; writingoriginal draft preparation, A.M.A. and C.M.K.; writing-review and editing, all authors; supervision, E.O. and C.M.K. All authors read and approved the final manuscript."

- Acknowledgment: "Thanks to BioRender.com, all illustrations in this article were created. The authors would like to thank Ms. Tan Shi for taking the sumac plant photo and Ms. Henriette Dietrich for the technical support."

\section{References}

1. van Duyn MAS, Pivonka E. Overview of the health benefits of fruit and vegetable consumption for the dietetics professional. J. Am. Diet. Assoc. 2000;100:1511-21. doi:10.1016/S0002-8223(00)00420-X.

2. Nordberg J, Arnér ESJ. Reactive oxygen species, antioxidants, and the mammalian thioredoxin system. Free Radic. Biol. Med. 2001;31:1287-312. doi:10.1016/S0891-5849(01)00724-9.

3. Burke-Gaffney A, Callister MEJ, Nakamura H. Thioredoxin: Friend or foe in human disease? Trends Pharmacol. Sci. 2005;26:398-404. doi:10.1016/j.tips.2005.06.005.

4. Clark JH. Green chemistry: Challenges and opportunities. Green Chem. 1999;1:1-8. doi:10.1039/a807961g. 
5. Clark JH. Green chemistry: Today (and tomorrow). Green Chem. 2006;8:17-21. doi:10.1039/B516637N.

6. Clark JH, Budarin V, Deswarte FEl, Hardy JJE, Kerton FM, Hunt AJ, et al. Green chemistry and the biorefinery: A partnership for a sustainable future. Green Chem. 2006;8:853. doi:10.1039/b604483m.

7. Mestres R. A brief structured view of green chemistry issues. Green Chem. 2004;6:G10. doi:10.1039/b314467b.

8. Tang SLY, Smith RL, Poliakoff M. Principles of green chemistry: Productively. Green Chem. 2005;7:761. doi:10.1039/b513020b.

9. Cacace JE, Mazza G. Mass transfer process during extraction of phenolic compounds from milled berries. J. Food Eng. 2003;59:379-89. doi:10.1016/S0260-8774(02)00497-1.

10. Rayne S, Mazza G. Biological activities of extracts from sumac (Rhus spp.): A review. Nat. Prec. 2007. doi:10.1038/npre.2007.631.1.

11. Cao J, Cao J, Wang H, Chen L, Cao F, Su E. Solubility improvement of phytochemicals using (natural) deep eutectic solvents and their bioactivity evaluation. J. Mol. Liq. 2020;318:113997. doi:10.1016/j.molliq.2020.113997.

12. Abraham AM, Alnemari RM, Jacob C, Keck CM. PlantCrystals-Nanosized plant material for improved bioefficacy of medical plants. Materials. 2020;13:4368. doi:10.3390/ma13194368.

13. Yassin DA, Nasim MJ, Abraham AM, Keck CM, Jacob C. Upcycling culinary organic waste: Production of plant particles from potato and carrot peels to improve antioxidative capacity. Current Nutraceuticals 2020. doi:10.2174/2665978601999200925163905.

14. Griffin S, Sarfraz M, Farida V, Nasim MJ, Ebokaiwe AP, Keck CM, Jacob C. No time to waste organic waste: Nanosizing converts remains of food processing into refined materials. J. Environ. Manage. 2018;210:114-21. doi:10.1016/j.jenvman.2017.12.084.

15. Georget E, Miller B, Callanan M, Heinz V, Mathys A. (Ultra) high pressure homogenization for continuous high pressure sterilization of pumpable foods - A review. Front. Nutr. 2014;1:15. doi:10.3389/fnut.2014.00015.

16. Velázquez-Estrada RM, Hernández-Herrero MM, Rüfer CE, Guamis-López B, Roig-Sagués AX. Influence of ultra high pressure homogenization processing on bioactive compounds and antioxidant activity of orange juice. Innov. Food Sci. Emerg. Technol. 2013;18:89-94. doi:10.1016/j.ifset.2013.02.005.

17. Saldo J, Suárez-Jacobo Á, Gervilla R, Guamis B, Roig-Sagués AX. Use of ultra-high-pressure homogenization to preserve apple juice without heat damage. High. Press. Res. 2009;29:52-6. doi:10.1080/08957950802715112.

18. Griffin S, Alkhayer R, Mirzoyan S, Turabyan A, Zucca P, Sarfraz M, et al. Nanosizing Cynomorium: Thumbs up for potential antifungal applications. Inventions. 2017;2:24. doi:10.3390/inventions2030024.

19. Abraham AM, Alnemari RM, Brüßler J, Keck CM. Improved antioxidant capacity of black tea waste utilizing PlantCrystals. Molecules. 2021;26:592. doi:10.3390/molecules26030592. 
20. Sarfraz M, Griffin S, Gabour Sad T, Alhasan R, Nasim MJ, Irfan Masood M, et al. Milling the mistletoe: Nanotechnological conversion of African mistletoe (Loranthus micranthus) intoantimicrobial materials. Antioxidants 2018. doi:10.3390/antiox7040060.

21. Pelikh O, Hartmann SF, Abraham AM, Keck CM. Nanocrystals for dermal application. In: Cornier J, Keck CM, van Voorde M de, editors. Nanocosmetics: From ideas to products. Cham, Switzerland: Springer; 2019. p. 161-177. doi:10.1007/978-3-030-16573-4_8.

22. Morshedloo MR, Maggi F, Tavakoli Neko H, Soleimani Aghdam M. Sumac (Rhus coriaria L.) fruit: Essential oil variability in Iranian populations. Ind. Crops Prod. 2018;111:1-7. doi:10.1016/j.indcrop.2017.10.002.

23. Beretta G, Rossoni G, Santagati NA, Facino RM. Anti-ischemic activity and endothelium-dependent vasorelaxant effect of hydrolysable tannins from the leaves of Rhus coriaria (Sumac) in isolated rabbit heart and thoracic aorta. Planta Med. 2009;75:1482-8. doi:10.1055/s-0029-1185797.

24. Candan F, Sökmen A. Effects of Rhus coriaria $L$ (Anacardiaceae) on lipid peroxidation and free radical scavenging activity. Phytother. Res. 2004;18:84-6. doi:10.1002/ptr.1228.

25. Candan F. Effect of Rhus coriaria L. (Anacardiaceae) on superoxide radical scavenging and xanthine oxidase activity. J. Enzyme Inhib. Med. Chem. 2003;18:59-62. doi:10.1080/1475636031000069273.

26. Sakhr K, El Khatib S. Physiochemical properties and medicinal, nutritional and industrial applications of Lebanese Sumac (Syrian sumac - Rhus coriaria): A review. Heliyon. 2020;6:e03207. doi:10.1016/j.heliyon.2020.e03207.

27. Alsamri H, Athamneh K, Pintus G, Eid AH, Iratni R. Pharmacological and antioxidant activities of Rhus coriaria L. (Sumac). Antioxidants 2021. doi:10.3390/antiox10010073.

28. Kosar M, Bozan B, Temelli F, Baser KHC. Antioxidant activity and phenolic composition of sumac (Rhus coriaria L.) extracts. Food Chem. 2007;103:952-9. doi:10.1016/j.foodchem.2006.09.049.

29. Capcarova M, Slamecka J, Abbas K, Kolesarova A, Kalafova A, Valent M, et al. Effects of dietary inclusion of Rhus coriaria on internal milieu of rabbits. J. Anim. Physiol. Anim. Nutr. 2012;96:45965. doi:10.1111/j.1439-0396.2011.01164.x.

30. Abu-Reida IM, Jamous RM, Ali-Shtayeh MS. Phytochemistry, pharmacological properties and industrial applications of Rhus coriaria L. (Sumac). Jordan J. Biol. Sci. 2014;7:233-44. doi:10.12816/0008245.

31. Chaves N, Santiago A, Alías JC. Quantification of the antioxidant activity of plant extracts: Analysis of sensitivity and hierarchization based on the method used. Antioxidants 2020. doi:10.3390/antiox9010076.

32. Huang D, Ou B, Prior RL. The chemistry behind antioxidant capacity assays. J. Agric. Food Chem. 2005;53:1841-56. doi:10.1021/jf030723c.

33. Cao G, Alessio HM, Cutler RG. Oxygen-radical absorbance capacity assay for antioxidants. Free Radic. Biol. Med. 1993;14:303-11. doi:10.1016/0891-5849(93)90027-R.

34. Middelberg APJ. Process-scale disruption of microorganisms. Biotechnol. Adv. 1995;13:491-551. doi:10.1016/0734-9750(95)02007-P. 
35. Jacobo ÁS, Saldo J, Gervilla R. Influence of high-pressure and ultra-high-pressure homogenization on antioxidants in fruit juice. In: Processing and impact on antioxidants in beverages. Oxford UK: Academic Press; 2014. p. 185-193. doi:10.1016/B978-0-12-404738-9.00019-2.

36. http://gaulinhomogenizer.com/.

37. Schilling S, Schmid S, Jäger H, Ludwig M, Dietrich H, Toepfl S, et al. Comparative study of pulsed electric field and thermal processing of apple juice with particular consideration of juice quality and enzyme deactivation. J. Agric. Food Chem. 2008;56:4545-54. doi:10.1021/jf0732713.

38. Saldo J, Suárez-Jacobo Á, Gervilla R, Guamis B, Roig-Sagués AX. Use of ultra-high-pressure homogenization to preserve apple juice without heat damage. High. Press. Res. 2009;29:52-6. doi:10.1080/08957950802715112.

39. Vinson JA, Su X, Zubik L, Bose P. Phenol antioxidant quantity and quality in foods: Fruits. J. Agric. Food Chem. 2001;49:5315-21. doi:10.1021/jf0009293.

40. Abu-Reidah IM, Ali-Shtayeh MS, Jamous RM, Arráez-Román D, Segura-Carretero A. HPLC-DAD-ESIMS/MS screening of bioactive components from Rhus coriaria L. (Sumac) fruits. Food Chem. 2015;166:179-91. doi:10.1016/j.foodchem.2014.06.011.

41. Braune A, Blaut M. Bacterial species involved in the conversion of dietary flavonoids in the human gut. Gut Microbes. 2016;7:216-34. doi:10.1080/19490976.2016.1158395.

42. Kawabata K, Yoshioka Y, Terao J. Role of intestinal microbiota in the bioavailability and physiological functions of dietary polyphenols. Molecules 2019. doi:10.3390/molecules24020370.

43. Pei R, Liu X, Bolling B. Flavonoids and gut health. Curr. Opin. Biotechnol. 2020;61:153-9. doi:10.1016/j.copbio.2019.12.018.

44. Thumann TA, Pferschy-Wenzig E-M, Moissl-Eichinger C, Bauer R. The role of gut microbiota for the activity of medicinal plants traditionally used in the European Union for gastrointestinal disorders. J. Ethnopharmacol. 2019;245:112153. doi:10.1016/j.jep.2019.112153.

45. Gil-Izquierdo A, Gil MI, Ferreres F. Effect of processing techniques at industrial scale on orange juice antioxidant and beneficial health compounds. J. Agric. Food Chem. 2002;50:5107-14. doi:10.1021/jf020162.

46. Jiménez N, Carrillo-Hormaza L, Pujol A, Álzate F, Osorio E, Lara-Guzman O. Antioxidant capacity and phenolic content of commonly used anti-inflammatory medicinal plants in Colombia. Ind. Crops Prod. 2015;70:272-9. doi:10.1016/j.indcrop.2015.03.050.

47. Griffin S, Sarfraz M, Hartmann SF, Pinnapireddy SR, Nasim MJ, Bakowsky U, et al. Resuspendable powders of lyophilized chalcogen particles with activity against microorganisms. Antioxidants 2018 . doi:10.3390/antiox7020023.

48. Keck CM. Particle size analysis of nanocrystals: Improved analysis method. Int. J. Pharm. 2010;390:3-12. doi:10.1016/j.ijpharm.2009.08.042.

49. Ordonez A, Gomez J, Vattuone M, Lsla M. Antioxidant activities of Sechium edule (Jacq.) Swartz extracts. Food Chem. 2006;97:452-8. doi:10.1016/j.foodchem.2005.05.024. 
50. Sharma OP, Bhat TK. DPPH antioxidant assay revisited. Food Chem. 2009;113:1202-5. doi:10.1016/j.foodchem.2008.08.008.

51. Katsube T, Tabata H, Ohta Y, Yamasaki Y, Anuurad E, Shiwaku K, Yamane Y. Screening for antioxidant activity in edible plant products: comparison of low-density lipoprotein oxidation assay, DPPH radical scavenging assay, and Folin-Ciocalteu assay. J. Agric. Food Chem. 2004;52:2391-6. doi:10.1021/jf035372g.

52. Ou B, Hampsch-Woodill M, Prior RL. Development and validation of an improved oxygen radical absorbance capacity assay using fluorescein as the fluorescent probe. J. Agric. Food Chem. 2001;49:4619-26. doi:10.1021/jf010586o.

53. Álvarez R, Carvalho CP, Sierra J, Lara O, Cardona D, Londoño-Londoño J. Citrus juice extraction systems: Effect on chemical composition and antioxidant activity of clementine juice. J. Agric. Food Chem. 2012;60:774-81. doi:10.1021/jf203353h.

\section{Figures}
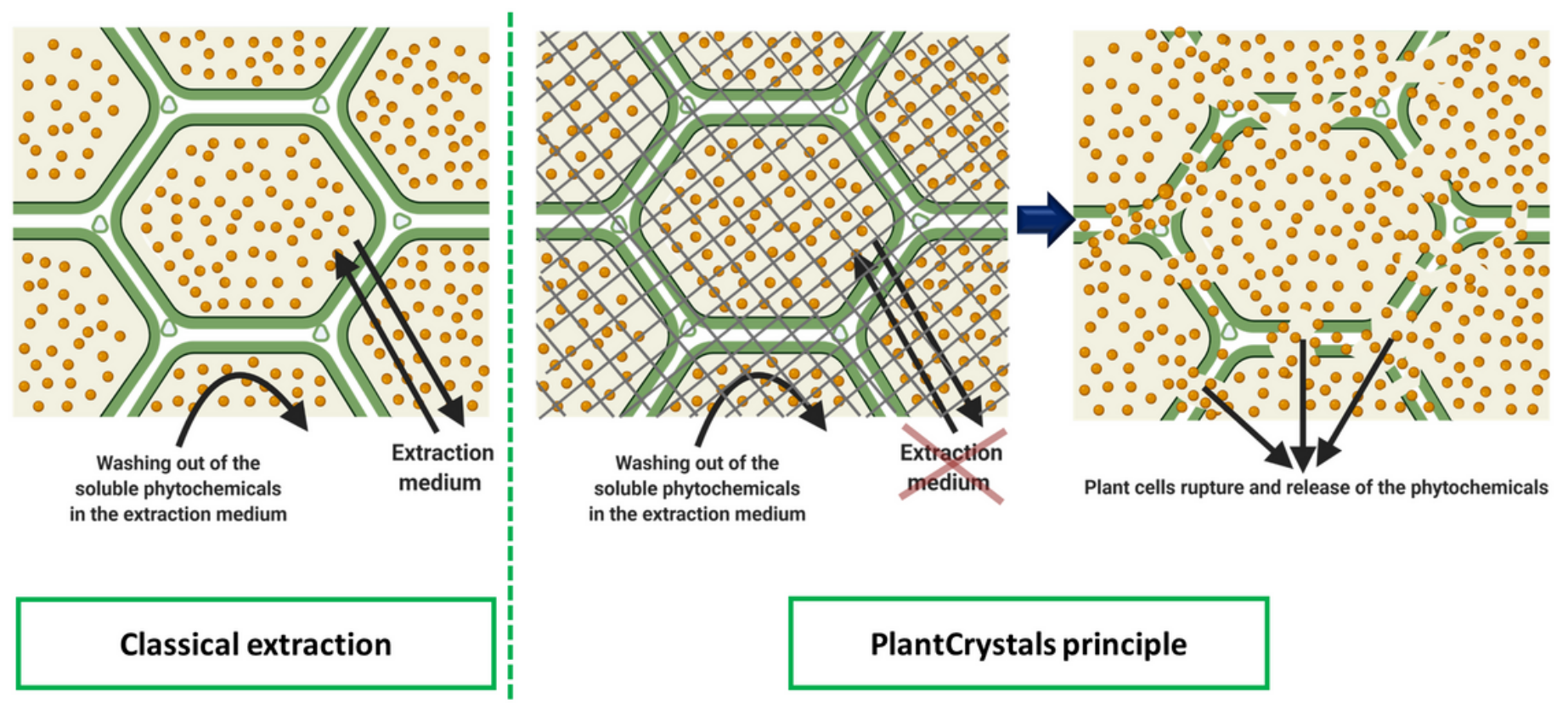
in the extraction medium

\section{PlantCrystals principle}

\section{Figure 1}

Comparison between the principle of the classical extraction process and PlantCrystal-technology. The green lines represent the plant cells and the yellow round particles represent the phytochemicals inside the plant cells. The grey net (in the middle image) represents the forces applied during HPH for plant cells rupture. 

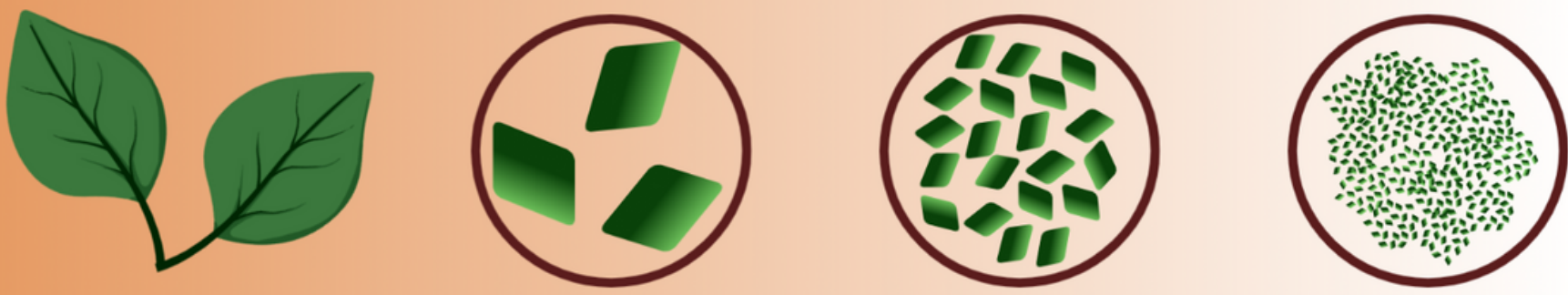

Dried plant

Ground bulk material

Micronized material
PlantCrystals size $<1 \mu \mathrm{m}$

Figure 2

Scheme of PlantCrystals production. Modified after [21].
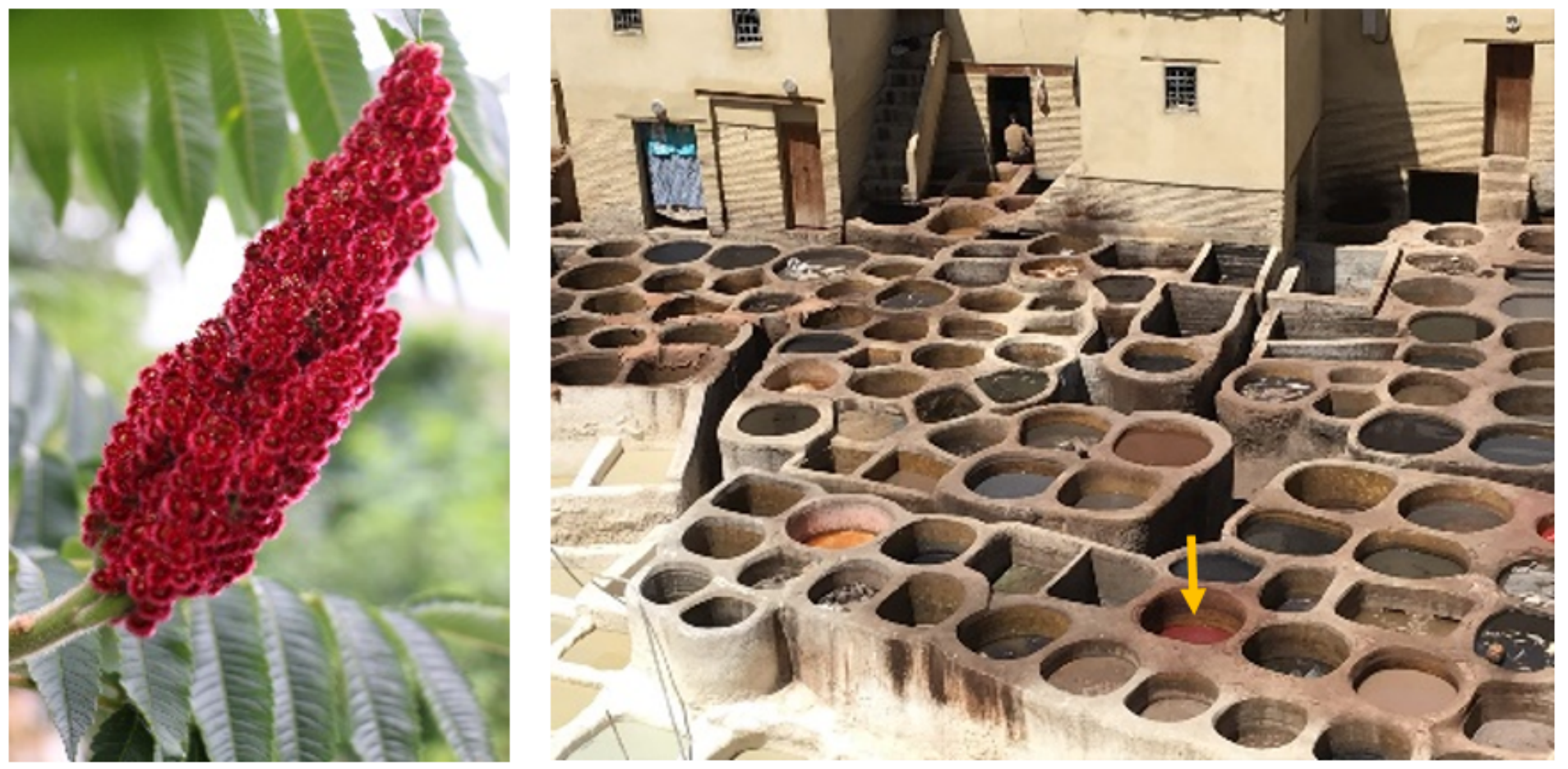

Figure 3

Sumac (Rhus coriaria L.) plant photo was taken in Marburg in July 2020 (left) and traditional tanneries of Fez Chouara in Morocco was taken in June 2019 (right). 


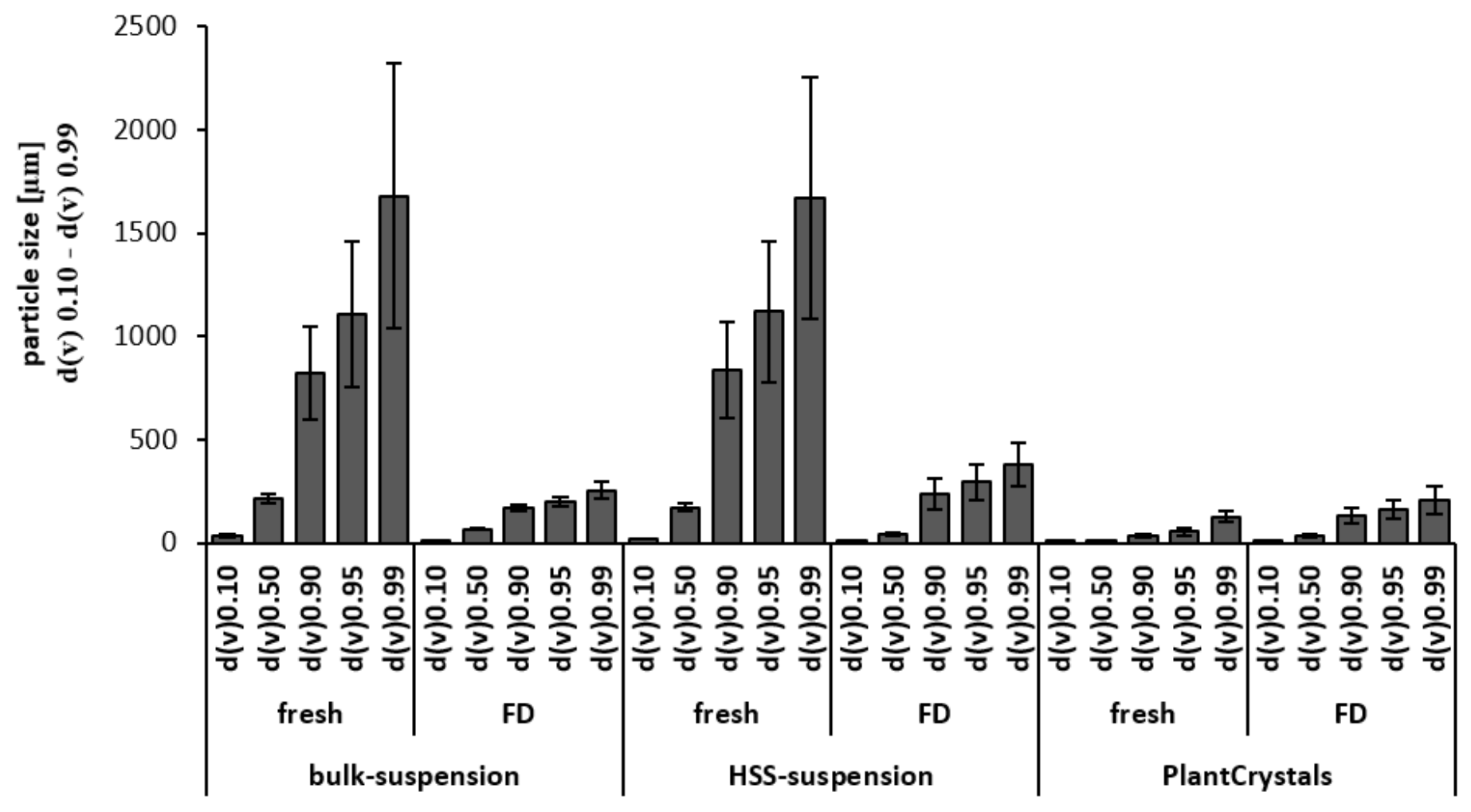

Figure 4

Laser diffraction data showed particle size of sumac suspensions before and after freeze drying (FD): bulk, HSS and HPH (PlantCrystals) suspensions. 


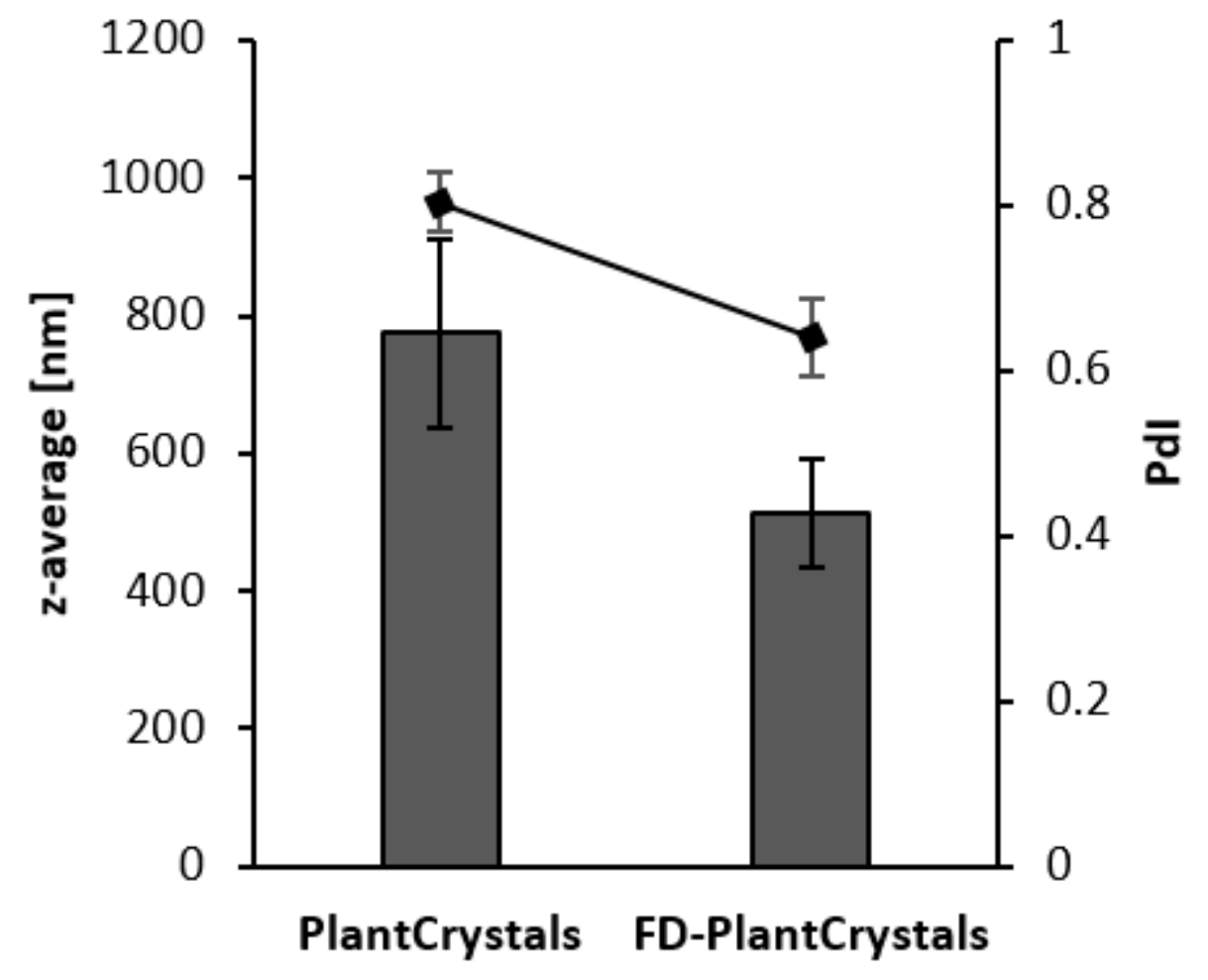

z-avg.

Figure 5

Dynamic light scattering (DLS) data show z-average and the polydispersity index (Pdl) of the produced PlantCrystals of sumac before and after freeze drying (FD).
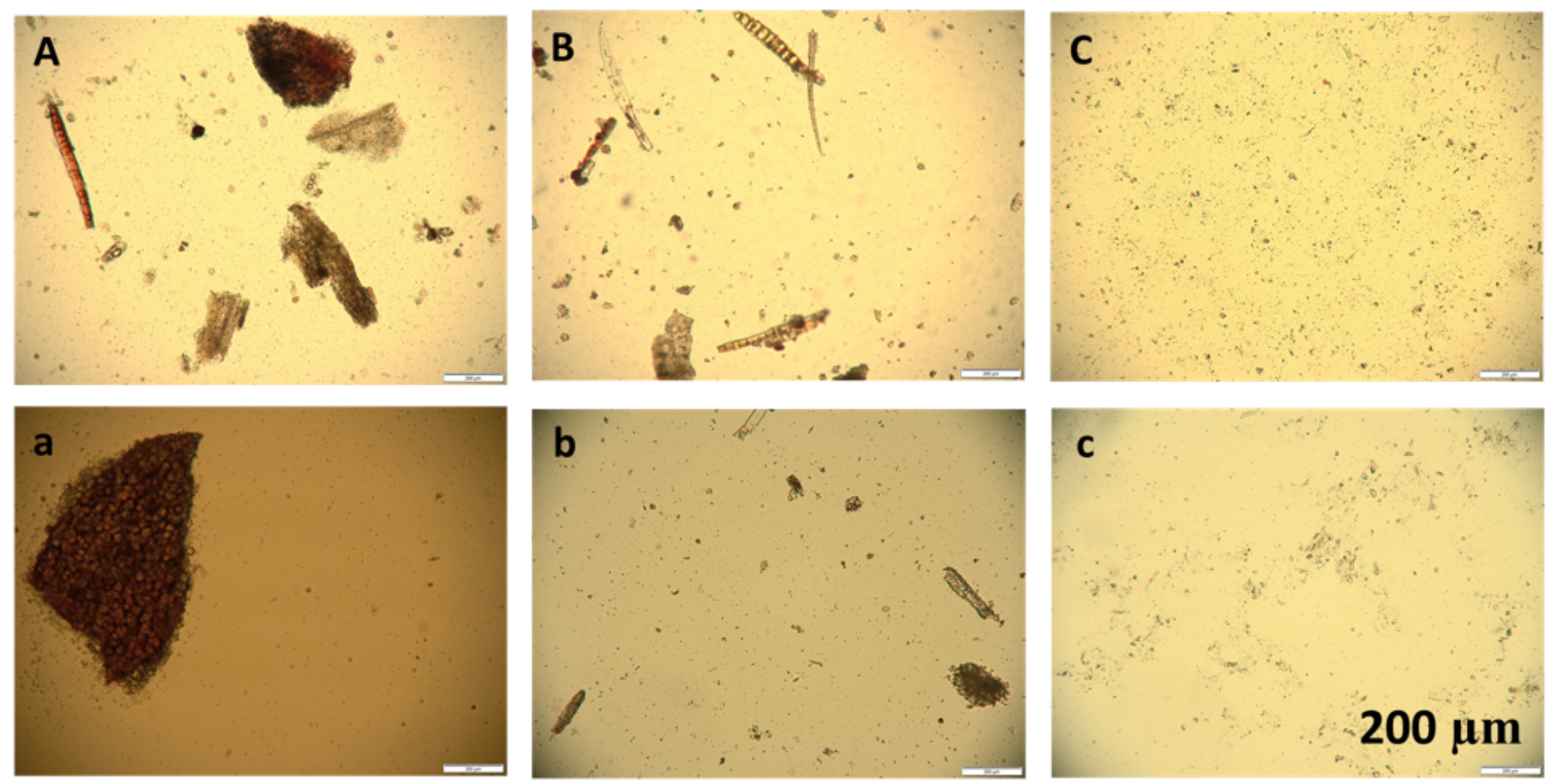

C 


\section{Figure 6}

Light microscopic images of: sumac fresh suspensions (upper panel): (A) bulk-material and its (B) HSSsuspension and (C) PlantCrystals after HPH; sumac freeze dried (FD, lower panel) suspensions (a) FDbulk-material and (b) FD-HSS-suspension (c) FD-PlantCrystals after re-dispersion (100-fold magnification and scale of $200 \mu \mathrm{m})$.

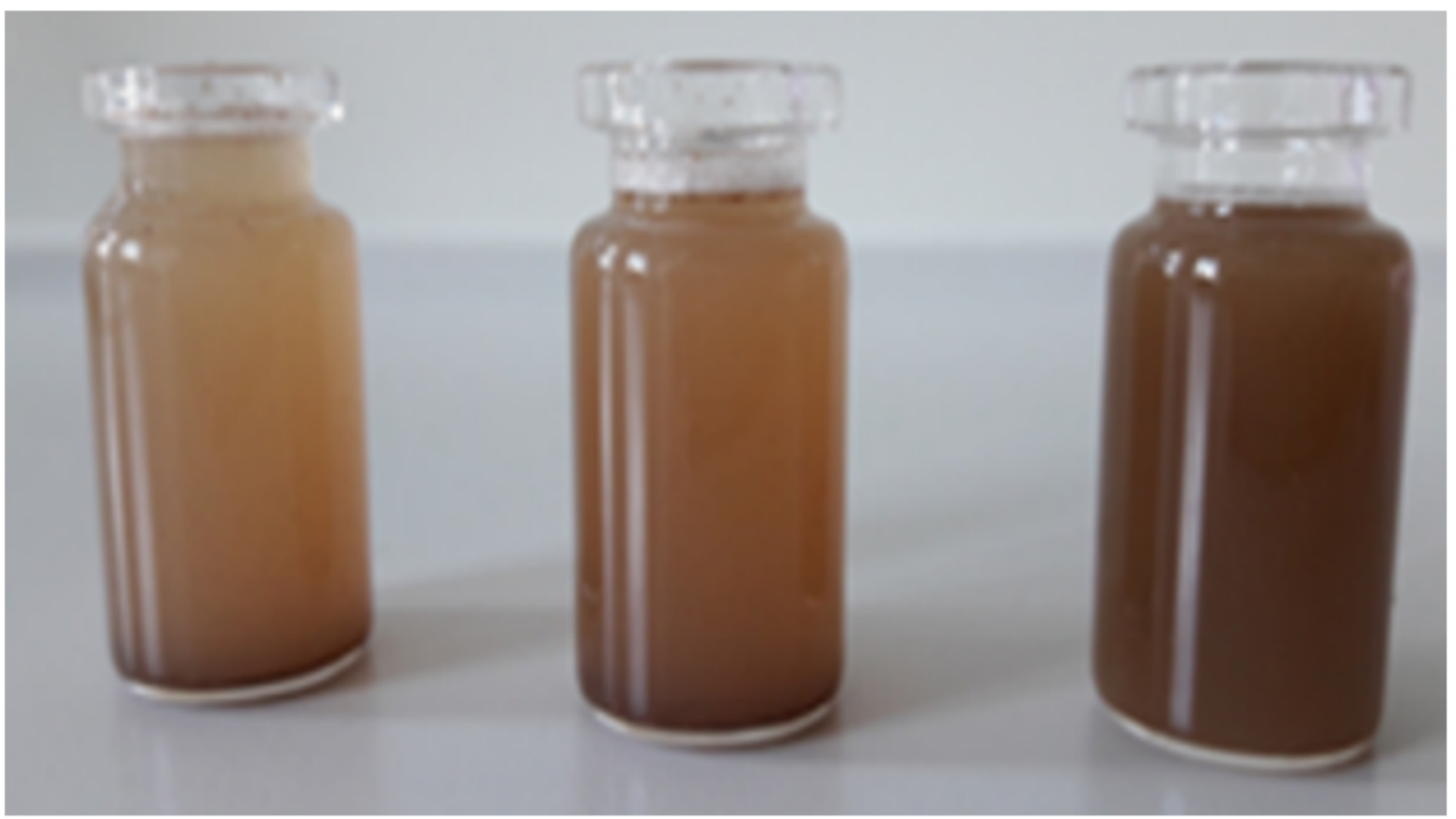

\section{Figure 7}

The produced fresh suspensions of sumac, from left to right: bulk, micron (HSS) and submicron (HPH) suspensions. 


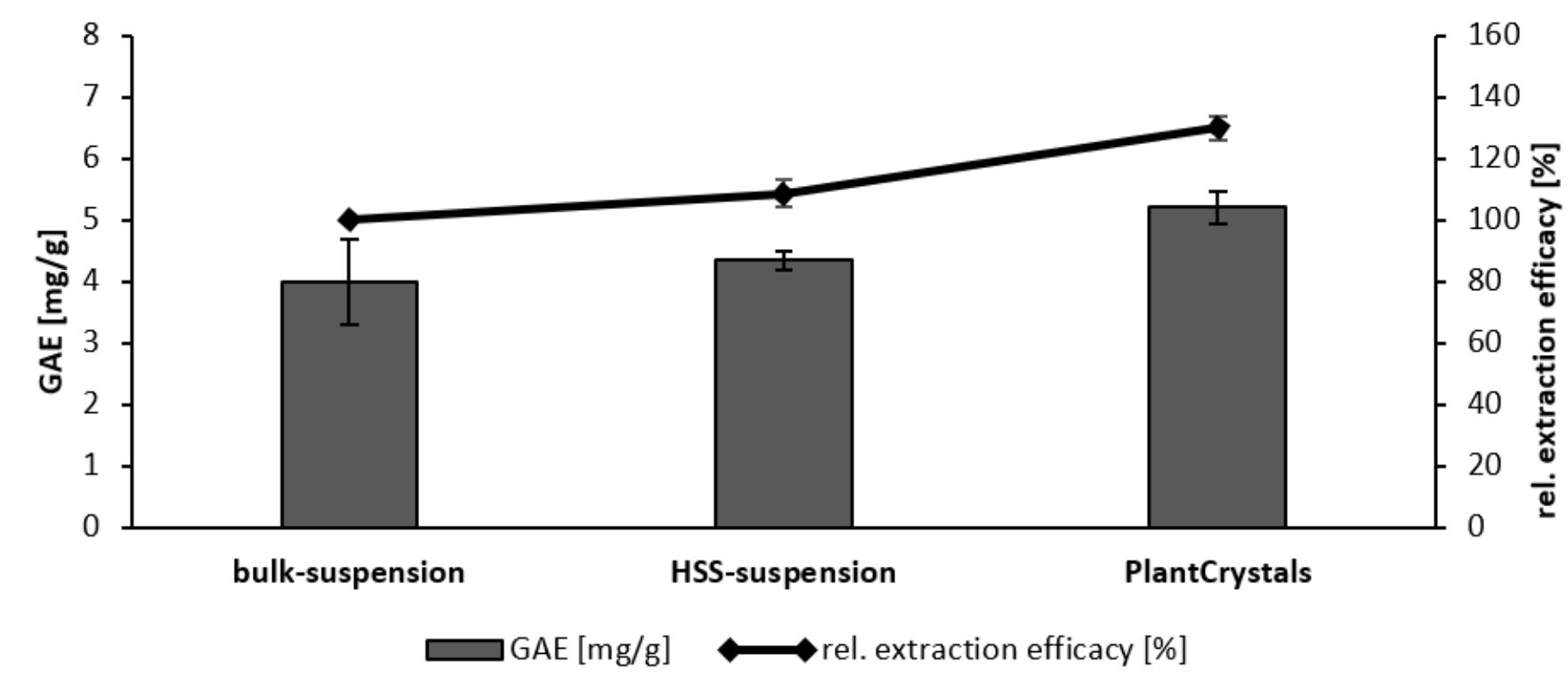

Figure 8

Determination of total polyphenol content of sumac suspensions as bulk materials, micronized (HSS) and HPH-suspension (PlantCrystals). Gallic acid was used as benchmark reference and the polyphenol content is expressed in gallic acid equivalents (GAE).

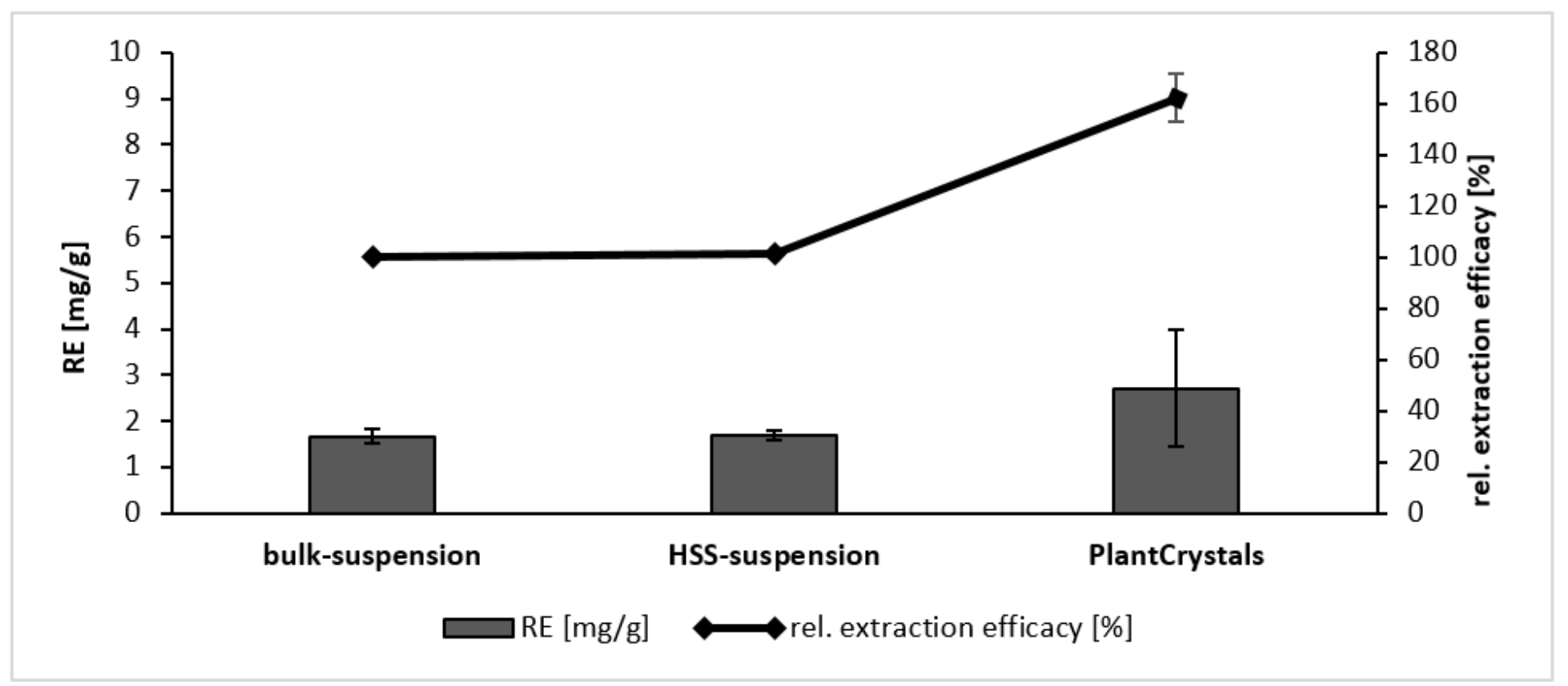

Figure 9

Determination of total flavonoid content of sumac suspensions as bulk materials, micronized (HSS) and $\mathrm{HPH}$-suspension (PlantCrystals). The flavonoid content is expressed as rutin equivalents (RE). Rutin was used as a benchmark control. 


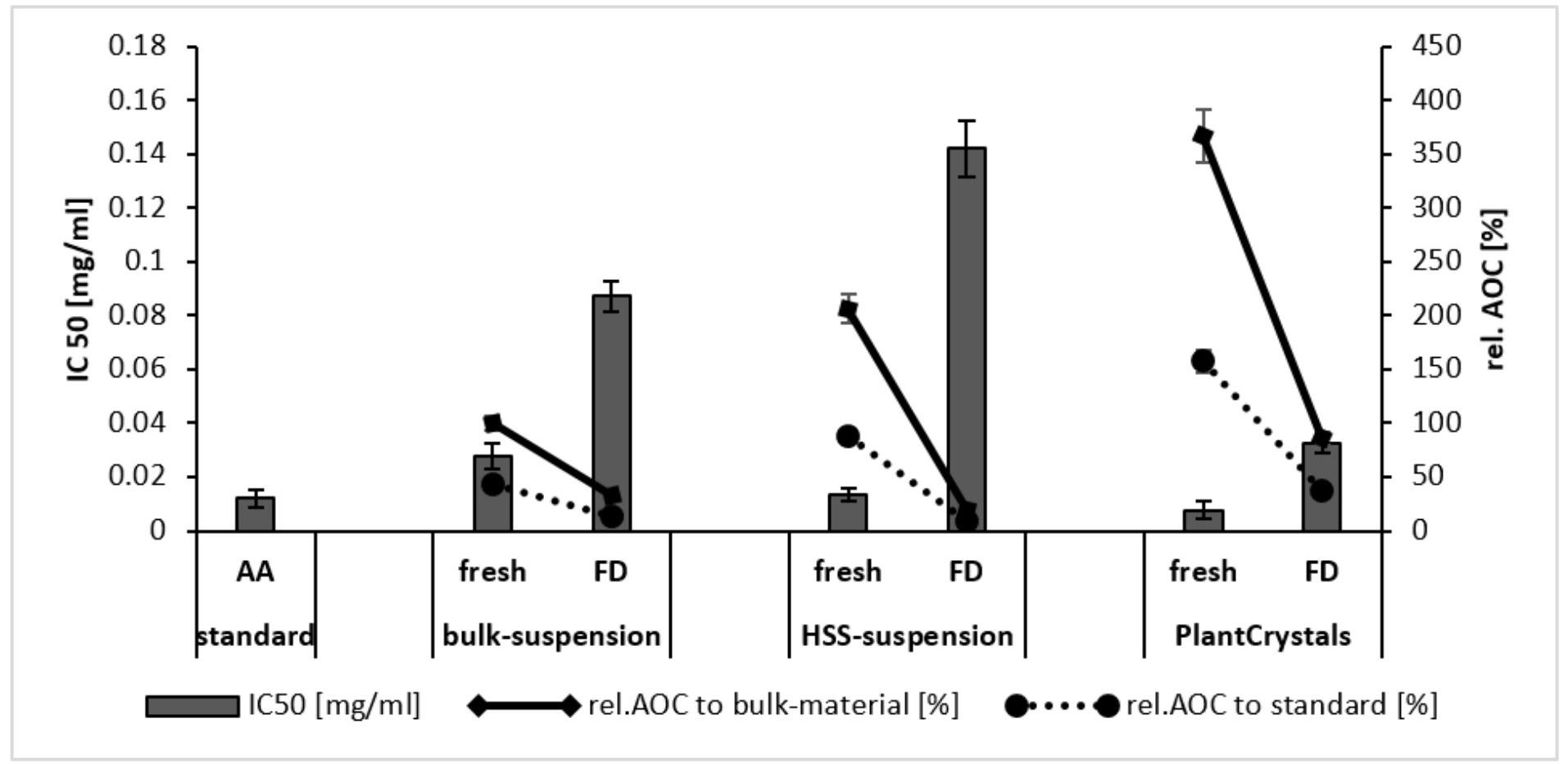

Figure 10

Antioxidant capacity using DPPH assay of sumac suspensions before and after freeze drying (FD) as bulk materials, micronized (HSS) and HPH-suspension (PlantCrystals). Their IC50 values were compared to the IC50 of AA (ascorbic acid), which was used as standard. No significant difference was noticed between the fresh PlantCrystals after $\mathrm{HPH}$ and the used standard.

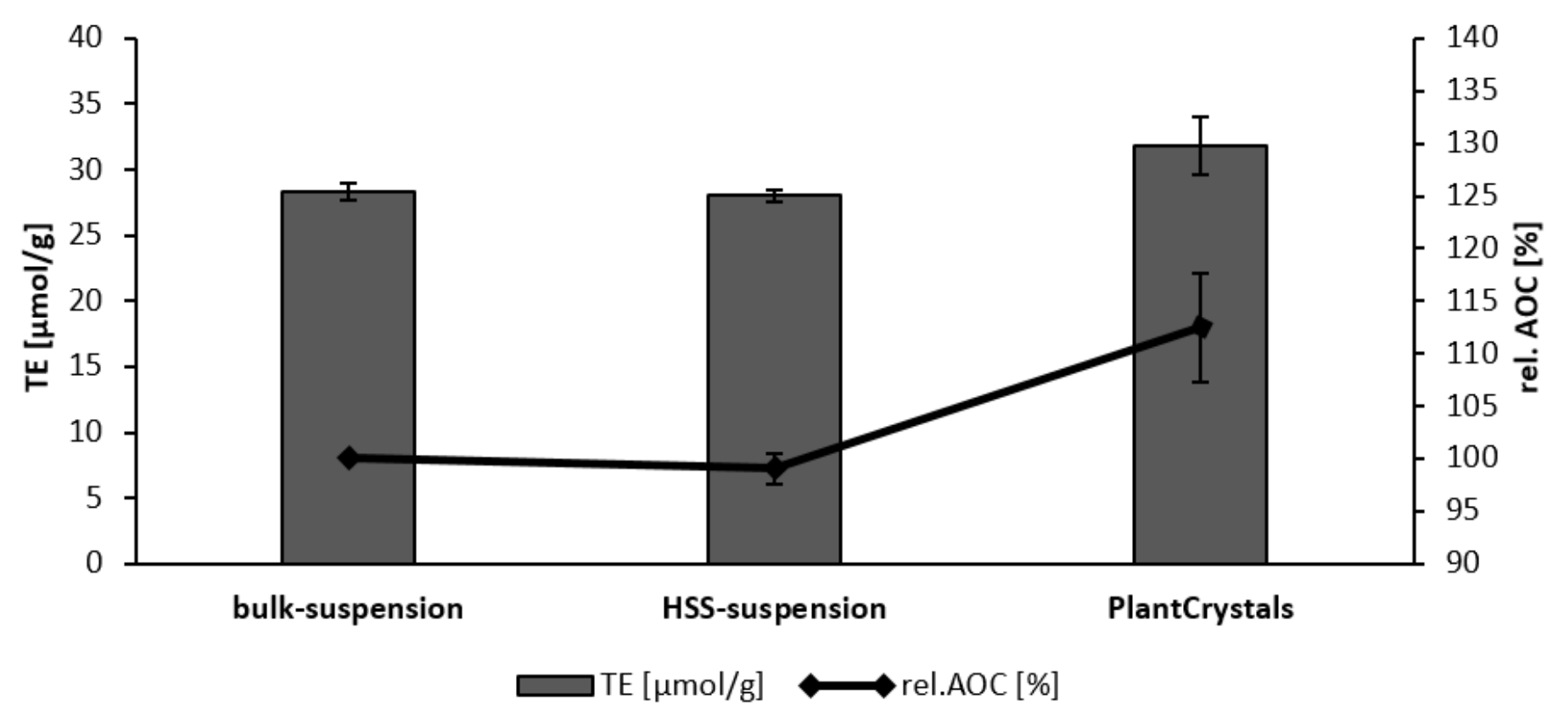

Figure 11 
Antioxidant capacity using FRAP assay of sumac suspensions as bulk materials, micronized (HSS) and $\mathrm{HPH}$-suspension (PlantCrystals).

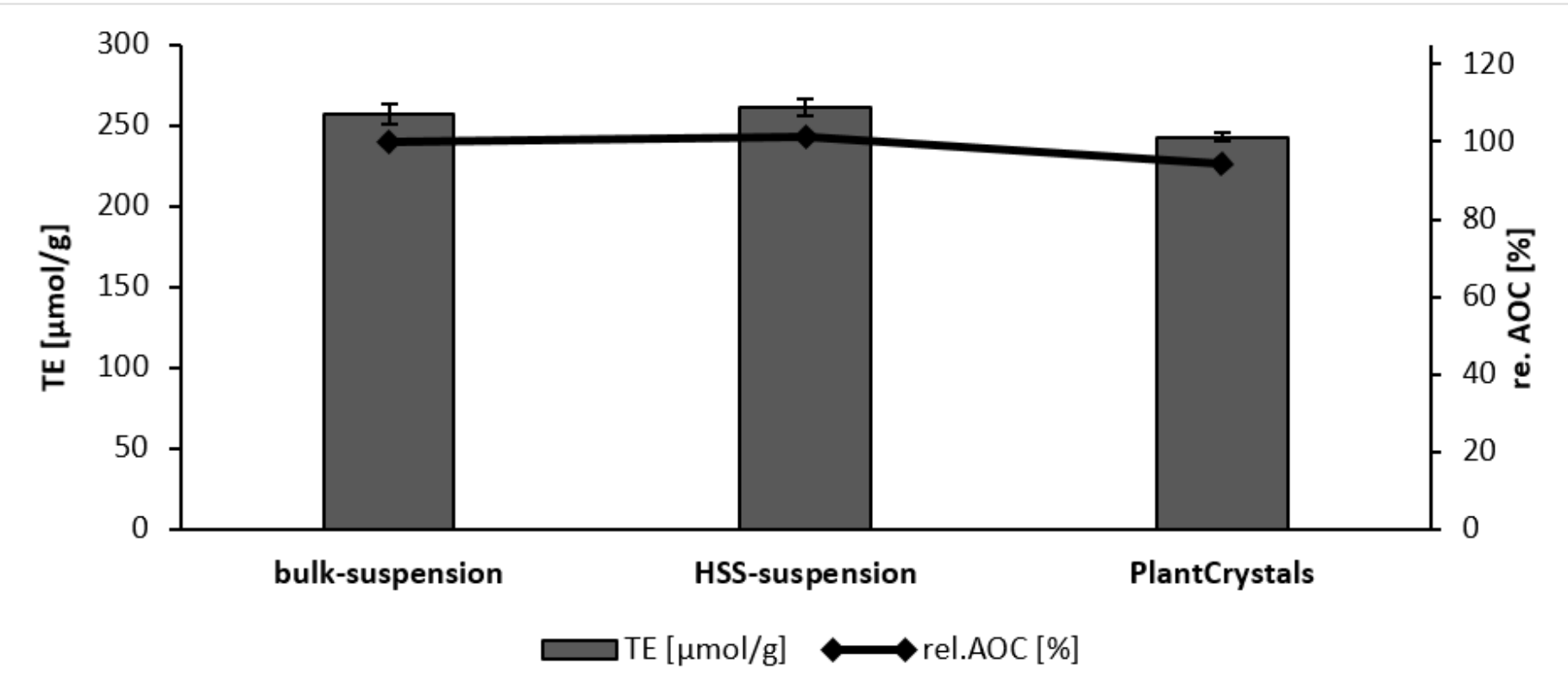

Figure 12

Antioxidant capacity using ABTS assay of sumac suspensions as bulk materials, micronized (HSS) and $\mathrm{HPH}$-suspension (PlantCrystals).

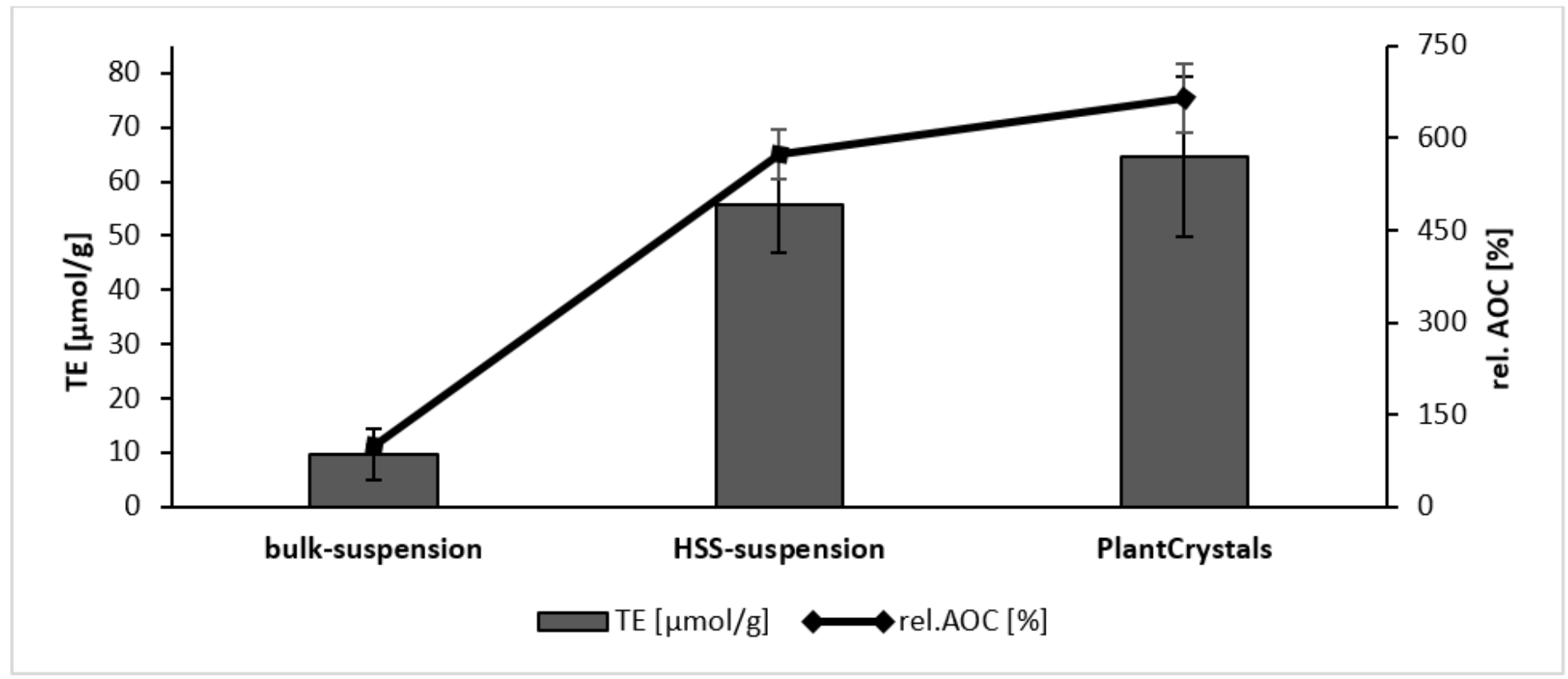

Figure 13

Antioxidant capacity using ORAC assay of sumac suspensions as bulk materials, micronized (HSS) and $\mathrm{HPH}$-suspension (PlantCrystals). ORAC value was expressed as $\mu \mathrm{mol}$ Trolox equivalent (TE) per $\mathrm{g}$ of the sample analyzed. 


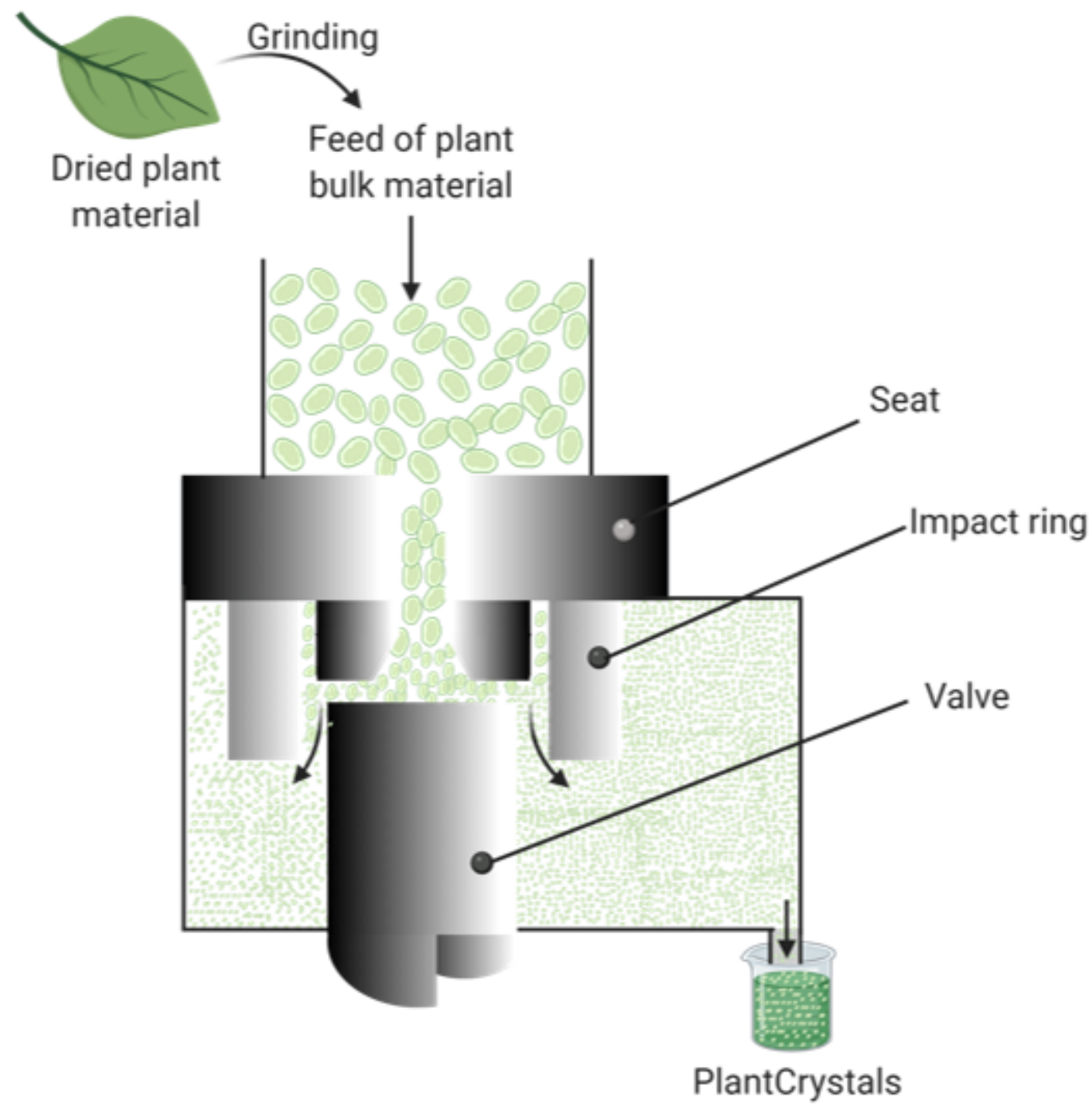

Figure 14

High pressure homogenization principle to produce PlantCrystals. Modified after [36]. 